\title{
Research on the Measuring System of Polymer-based Capacitive Micromachined Ultrasonic Transducer Array for Circular Planar Target
}

\author{
Gia Thinh Bui, 2, a, Yu-Hsuan Chiang ${ }^{1, b}$, Da-Chen Pang ${ }^{1, c^{*}}$ \\ ${ }^{1}$ Department of Mechanical Engineering, National Kaohsiung University of Applied Sciences, \\ 415 Jiangong Road, Sanmin District, Kaohsiung City 807, Taiwan (R.O.C.) \\ ${ }^{2}$ Department of Electrical and Mechanical Engineering, Hai Phong University, \\ 171 Phan Dang Luu Road, Kien An District, Hai Phong City, Vietnam.
}

abuigiathinh1784@gmail.com, ${ }^{b}$ molodychiang926@gmail.com, ${ }^{c}$ pang@kuas.edu.tw

Keywords: Polymers, Capacitive Micromachined Ultrasonic Transducer (CMUT), Four-quadrant array, Position detection, Circular target.

\begin{abstract}
The purpose of this research is using a polymer-based capacitive micro-machined ultrasonic transducer (CMUT) array to determine the position and dimension of circular planar target. The polymer-based CMUT is consist of an indium tin oxide-polyethylene terephthalate (ITO-PET) substrate and SU-8 sidewall and vibrating membranes. The CMUT has advantages of flexibility, low cost and large sensing area application comparing with silicon-based sensor. A fourquadrant (4Q) array is used to determine the position of the circular target based on the time of flight (TOF) and magnitude of the acoustic echo signals reflected from the target. The measuring system is designed to determine diameter and position of the transparent objects for pick and place on a conveyor. The four quadrant array has an overall dimension of $6 \mathrm{~mm} \times 6 \mathrm{~mm}$ and can detect target diameter up to $3.6 \mathrm{~mm}$ with a maximum eccentricity of $1.2 \mathrm{~mm}$. An algorithm is proposed to correct nonlinearity error when the target is away from the center of the four-quadrant array. The ultrasonic measuring system is constructed and tested to demonstrate its feasibility. The experimental results show the position error of $0.052 \mathrm{~mm}$ and dimension error of $0.42 \%$ in area at the worst scenario.
\end{abstract}

\section{Introduction}

Ultrasound is used to detect objects and measure its position without contact. There are many ultrasonic applications requiring position detection such as nondestructive testing, underwater sonar, and medical sonography. Haller and Khuri-Yakub developed the first capacitive micromachined ultrasonic transducer using silicon-based semiconductor technology [1]. The CMUT has advantages of miniaturization of devices, wide bandwidth, and high intensity focused ultrasound [2]. Later, Chang et al. introduced the first polymer-based CMUT using SU-8 photoresist for surgical imaging applications [3]. The polymer materials offer additional benefits such as flexibility, light transparency, and low material cost for disposable use. The polymer-based CMUT can be applied for large sensing area for example surface roughness measurement for sand papers [4].

Four quadrant detectors and laser beam are commonly used for detection of lateral displacement at $\mathrm{X}$ and $\mathrm{Y}$ dimensions and applied in alignment and tracking of optical systems [5-7]. The transfer function of four quadrant sensor detector and circular light profile is inherently nonlinear but around the detector center is linear approximately [8]. Dang et al. proposed a linearization method for photosensitive quadrant detector to expand linear interval and decrease nonlinear error so the linear range of detector is improved [9]. Mohammed and Rehman presented a four-quadrant capacitive angular position sensor which can cover full $360^{\circ}$ range for industrial robot applications [10].

The purpose of this research is to develop a measuring system using a polymer-based 4Q CMUT array for position and dimension measurement of transparent objects. The existing 4Q sensor has limitation on operating range because the nonlinear error will increase dramatically if the target moves away from the center. This study will purpose a simple correcting algorithm for improving 
operating range. Theoretical model and experiment testing will be conducted with different target diameters and eccentricities for verification. The 4Q CMUT array can be applied to its maximum range, the border of the sensor.

\section{Principle of Operation}

In this study, the polymer-based CMUT array is designed as $2 \times 2$ quadrants for non-contacting position detection. A single quadrant of CMUT has dimension of $3 \mathrm{~mm} \times 3 \mathrm{~mm}$ including 416 circular cells with membrane diameter of $143 \mu \mathrm{m}$. The four-quadrant CMUT design is shown in Fig. 1. The polymer-based CMUT array is made using ITO-PET substrate and SU-8 2002 photoresist material for sidewall and membrane, gold top electrode. Each CMUT cell has a membrane thickness of $5 \mu \mathrm{m}$, a golden top electrodes thickness of $0.2 \mu \mathrm{m}$, and sidewall height of $2 \mu \mathrm{m}$.

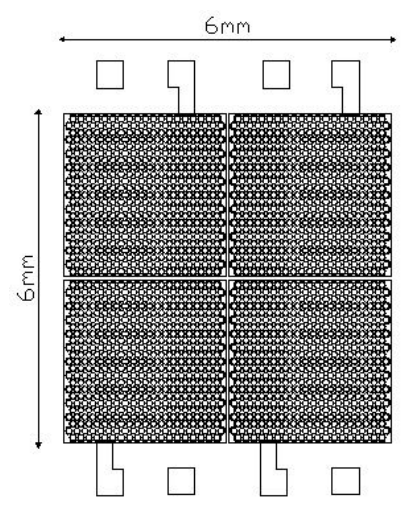

Fig. 1 Drawing of a four-quadrant CMUT array.

The position and dimension of the circular target can be determined by the magnitude of the acoustic echo signals reflected from the target to the four-quadrant CMUT array. The distance between target and CMUT can be calculated by time of flight between a transmission and reception. Assume that the surface of the circular planer target is placed in parallel with the surface of the 4Q CUMT. The position of the centroid of the target in the $\mathrm{X}-\mathrm{Y}$ plane is measured by the reflecting signals provided by the $4 \mathrm{Q}$ CMUT (referred to here as $\mathrm{Q}_{1}, \mathrm{Q}_{2}, \mathrm{Q}_{3}$, and $\mathrm{Q}_{4}$ ), and $\mathrm{r}$ is the eccentricity from the center of the $4 \mathrm{Q} C M U T$ array. The $\mathrm{X}$ and $\mathrm{Y}$ positioning signals of the measuring system are defined as $X_{Q}$ and $Y_{Q}$. The centroid of the target is calculated by reflecting signals provided by four quadrants and its geometric position as follows [4]:

$$
\begin{aligned}
& X_{Q}=\frac{\left(Q_{1}+Q_{4}\right)-\left(Q_{2}+Q_{3}\right)}{Q_{1}+Q_{2}+Q_{3}+Q_{4}}=1-\frac{2}{\pi}\left(\cos ^{-1}\left(\frac{X}{r}\right)-\frac{X}{r} \sqrt{1-\left(\frac{X}{r}\right)^{2}}\right) \\
& Y_{Q}=\frac{\left(Q_{1}+Q_{2}\right)-\left(Q_{3}+Q_{4}\right)}{Q_{1}+Q_{2}+Q_{3}+Q_{4}}=1-\frac{2}{\pi}\left(\cos ^{-1}\left(\frac{Y}{r}\right)-\frac{Y}{r} \sqrt{1-\left(\frac{Y}{r}\right)^{2}}\right)
\end{aligned}
$$

The positions of the circular target and 4Q CMUT array are shown in Fig. 2. The target will rotate with an eccentricity $r$ in counterclockwise. The ideal $\mathrm{X}$ and $\mathrm{Y}$ positions of target at a rotating angle $\theta$ in $\mathrm{XY}$ plane are measured as:

$$
\begin{gathered}
X_{\text {ideal }}=r \cos \theta \\
Y_{\text {ideal }}=r \sin \theta
\end{gathered}
$$




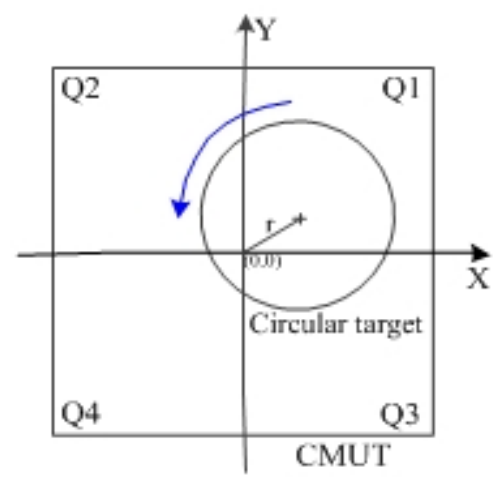

Fig. 2 Schematic of target position measurement using the 4Q CMUT.

There is a measurement error using four quadrant sensor if the target is away from the center of the sensor. The correcting factor $k$ is defined as:

$$
k=\frac{\sqrt{X_{\text {ideal }}^{2}+Y_{\text {ideal }}^{2}}}{\sqrt{X_{Q}^{2}+Y_{Q}^{2}}}
$$

Therefore, the $\mathrm{X}$ and $\mathrm{Y}$ positions after correcting measurement error can be described:

$$
\begin{aligned}
& X_{D}=k X_{Q} \\
& Y_{D}=k Y_{Q}
\end{aligned}
$$

The 4Q CMUT array has an overall dimension of $6 \mathrm{~mm} \times 6 \mathrm{~mm}$ and three different sizes of targets are selected with diameters of $3 \mathrm{~mm}, 3.3 \mathrm{~mm}$, and $3.6 \mathrm{~mm}$. Four different eccentricities, 0.3 $\mathrm{mm}, 0.6 \mathrm{~mm}, 0.9 \mathrm{~mm}$, and $1.2 \mathrm{~mm}$, are chosen to illustrate nonlinear measurement errors due to eccentricity. Fig. 3 shows the correcting factor $k$ from $0^{\circ}$ to $360^{\circ}$ at different eccentricities $r$ and angles $\theta$. Notice that if the target moves away from the center, the error correction is greater.

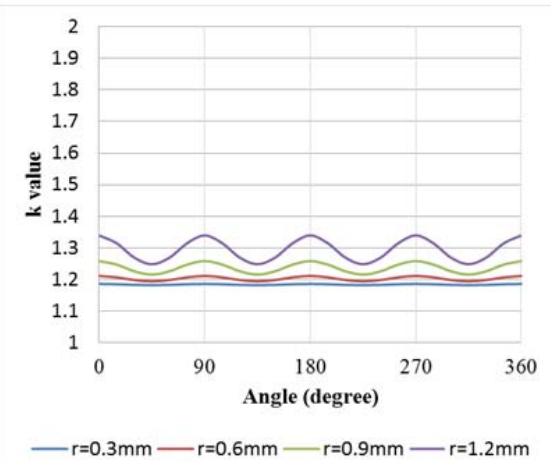

(a)

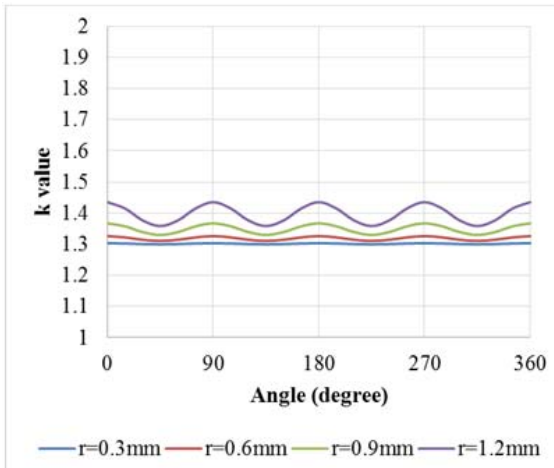

(b)

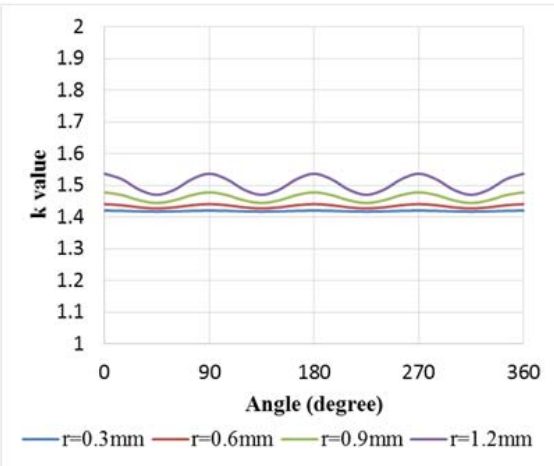

(c)

Fig. 3 Correction factors $k$ at different eccentricities $r$ and angles $\theta$.

(a) target diameter of $3.0 \mathrm{~mm}$, (b) target diameter of $3.3 \mathrm{~mm}$, (c) target diameter of $3.6 \mathrm{~mm}$.

\section{Experimental Results and Discussion}

In the experiments, the 4Q CMUT array is excited with a DC of $100 \mathrm{~V}$ and AC of $300 \mathrm{~V}$. The received signals are processed and the data is acquired using National Instruments PXI-5105 highspeed digitizers. This instrument can acquire multi-channel data therefor $\mathrm{X}$ and $\mathrm{Y}$ coordinate signal data are processed simultaneously to locate position of the circular plug via the LabVIEW software. Fig. 4 shows the schematic of experimental setup. 


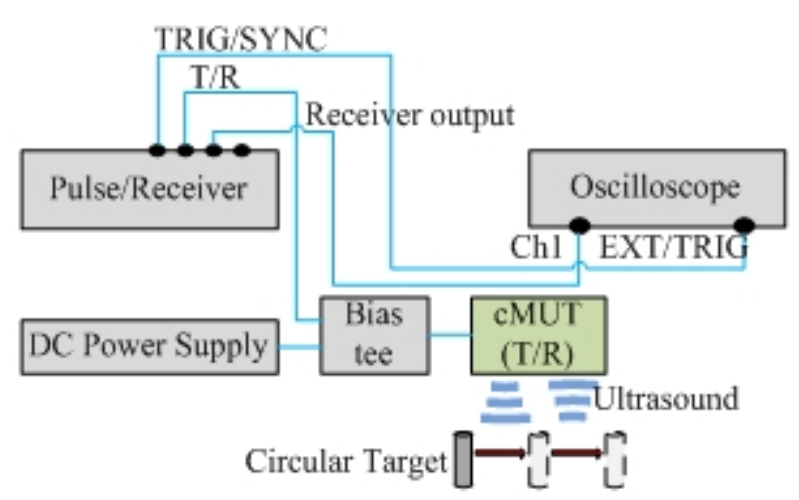

Fig. 4 Schematic of measuring system of $4 \mathrm{Q}$ CMUT array.

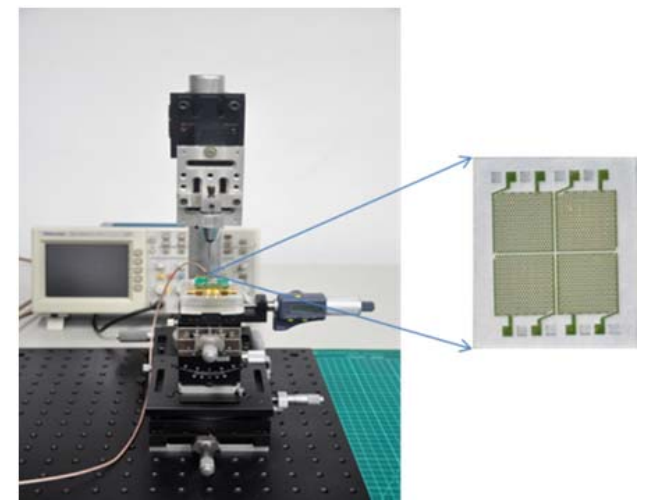

Fig. 5 Experimental setup of measuring system of 4Q CMUT array.

A cylinder stainless steel plug was used as the target in testing with different diameters of $3 \mathrm{~mm}$, $3.3 \mathrm{~mm}$ and $3.6 \mathrm{~mm}$. The 4Q CMUT is placed at a paralleled plane to the surface of target with distance of $10 \mathrm{~mm}$. The target is rotated counterclockwise $360^{\circ}$ with circular path paralleled the $4 \mathrm{Q}$ CMUT array as shown in Fig. 5. The target is measured with every $15^{\circ}$ angle. The experiments are repeated at different eccentricities of $0.3 \mathrm{~mm}, 0.6 \mathrm{~mm}, 0.9 \mathrm{~mm}$ and $1.2 \mathrm{~mm}$.

The ultrasonic reflecting signals of each quadrant are captured to measure $\mathrm{X}$ and $\mathrm{Y}$ positions of the target. Fig. 6 shows the reflecting signals of the 4Q CMUT array with target diameter of $3.6 \mathrm{~mm}$, eccentricity of $0.9 \mathrm{~mm}$, and angle of $45^{\circ}$. The amplitudes of received signals from quadrant $1,2,3$, and 4 are $45.492 \mathrm{mV}, 13.681 \mathrm{mV}, 31.349 \mathrm{mV}$, and $64.055 \mathrm{mV}$. The LabVIEW software is used to calculate position and dimension of the target. The dimension error for three target diameters is $0.043 \mathrm{~mm}^{2}$ or $0.42 \%$ in area.

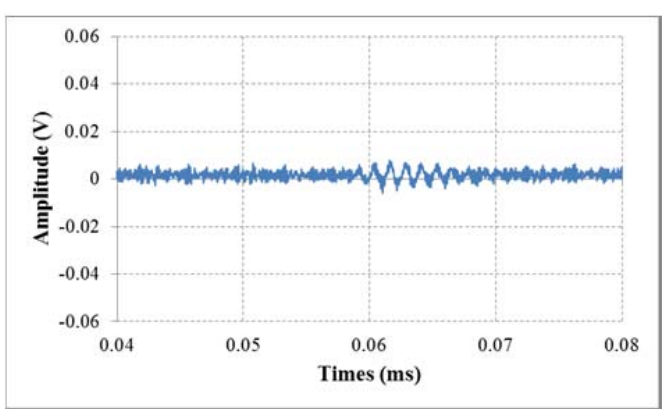

$\mathrm{Q}_{2}=13.681 \mathrm{mV}$

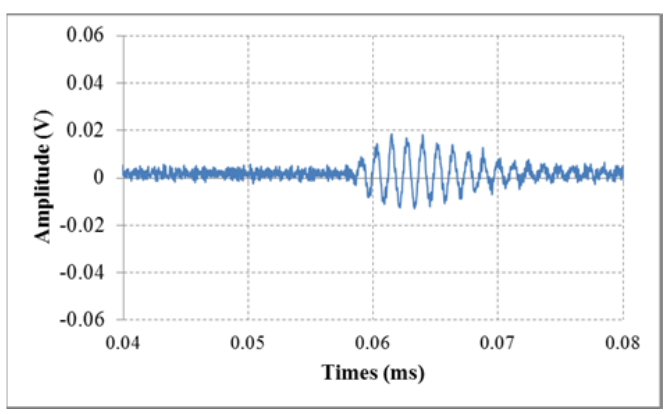

$\mathrm{Q}_{3}=31.349 \mathrm{mV}$
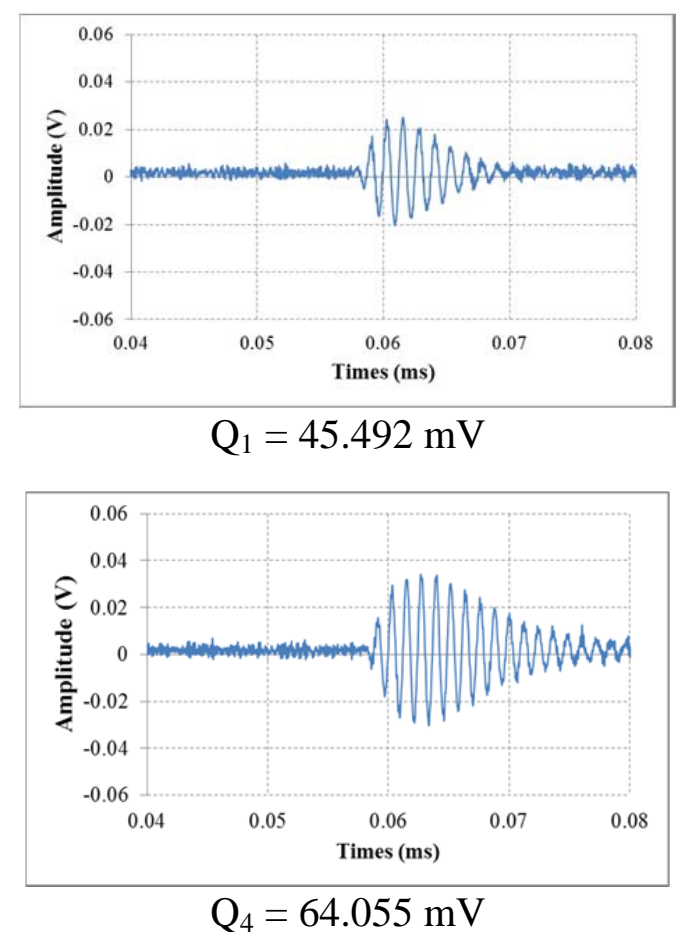

Fig. 6 Ultrasonic reflecting signals of the 4Q CMUT array.

The measuring positions of target in experiments are not perfect. Position errors of $X_{D}$ and $Y_{D}$ are given in Eq. (8) and Eq. (9), where $N$ is the number of experiments. 


$$
\begin{gathered}
\sigma_{X}=\sqrt{\frac{\sum\left(X_{\text {ideal }}-X_{D}\right)^{2}}{N-1}} \\
\sigma_{Y}=\sqrt{\frac{\sum\left(Y_{\text {ideal }}-Y_{D}\right)^{2}}{N-1}}
\end{gathered}
$$

The experimental results for position measurement are shown in Fig. 7, Fig. 8 and Fig. 9. Three cases with the target diameters of $3 \mathrm{~mm}, 3.3 \mathrm{~mm}$ and $3.6 \mathrm{~mm}$ are illustrated. Each target has four different eccentricities of $0.3 \mathrm{~mm}, 0.6 \mathrm{~mm}, 0.9 \mathrm{~mm}$, and $1.2 \mathrm{~mm}$, respectively. The correcting factor $k$ value increases when the target diameter increases. Also, the $k$ value increases as the eccentricity increases. It indicates that the error is less effective for smaller target and smaller eccentricity.

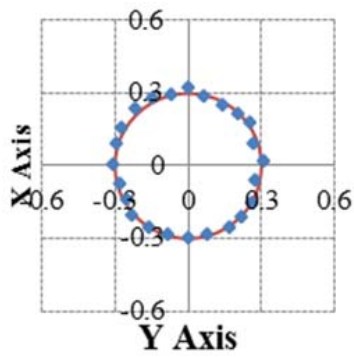

(a)

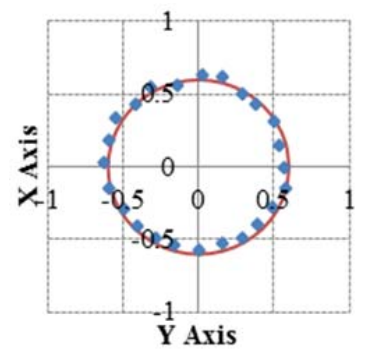

(b)

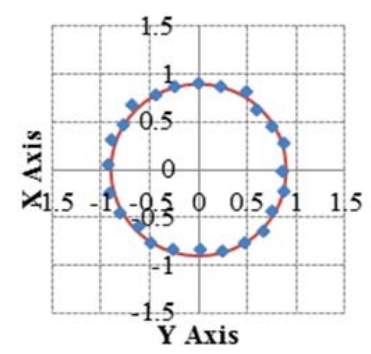

(c)

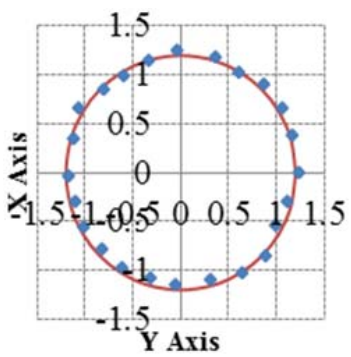

(d)

Solid line: ideal circular orbits. Dot point: experimental results

Fig. 7 Comparisons between experimental results and ideal conditions for circular orbits with target diameter of $3.0 \mathrm{~mm}$ at eccentricities of (a) $0.3 \mathrm{~mm}$, (b) $0.6 \mathrm{~mm}$, (c) $0.9 \mathrm{~mm}$, (d) $1.2 \mathrm{~mm}$.

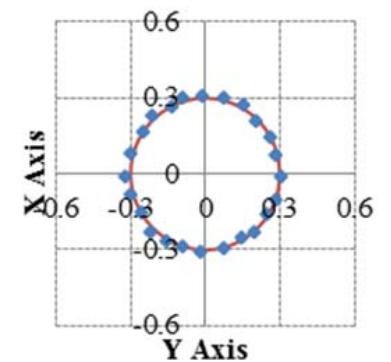

(a)

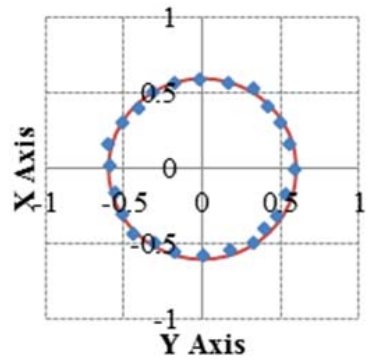

(b)

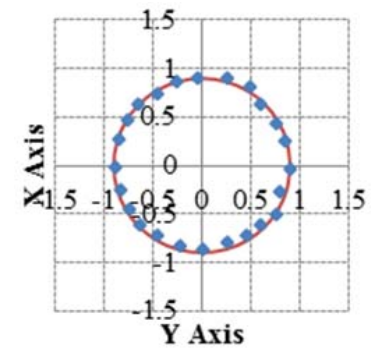

(c)

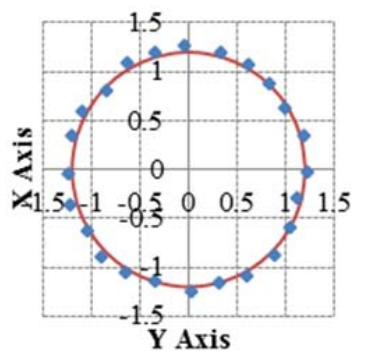

(d)

Solid line: ideal circular orbits. Dot point: experimental results

Fig. 8 Comparisons between experimental results and ideal conditions for circular orbits with target diameter of $3.3 \mathrm{~mm}$ at eccentricities of (a) $0.3 \mathrm{~mm}$, (b) $0.6 \mathrm{~mm}$, (c) $0.9 \mathrm{~mm}$, (d) $1.2 \mathrm{~mm}$.

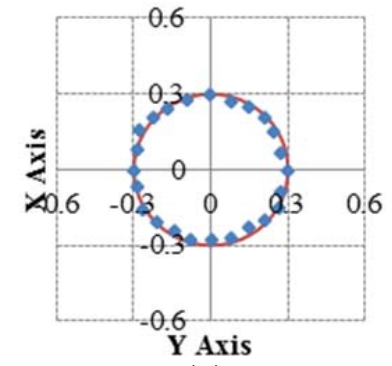

(a)

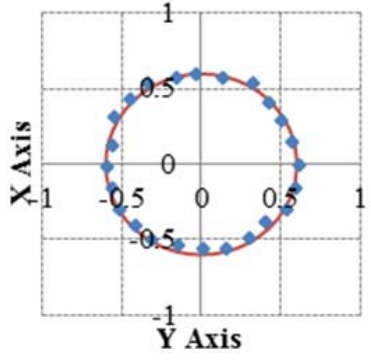

(b)

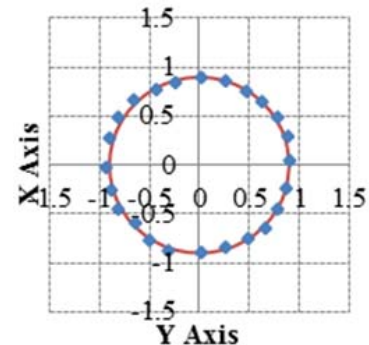

(c)

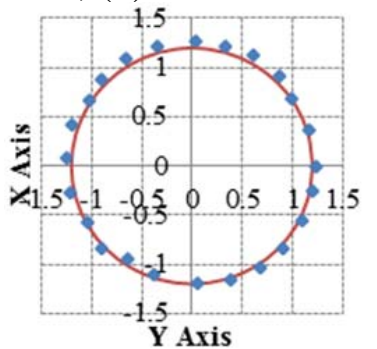

(d)

Solid line: ideal circular orbits. Dot point: experimental results

Fig. 9 Comparisons between experimental results and ideal conditions for circular orbits with target diameter of $3.6 \mathrm{~mm}$ at eccentricities of (a) $0.3 \mathrm{~mm}$, (b) $0.6 \mathrm{~mm}$, (c) $0.9 \mathrm{~mm}$, (d) $1.2 \mathrm{~mm}$.

Table 1 summaries the experimental errors for different target diameters OD and eccentricities $r$. The correcting factor $k$ is applied based on the eccentricity. There are a total of 25 points measured 
for each circle. The experiment errors are calculated and compared to inherited theoretical errors from eccentricity correction. There is no experiment test at the center (zero eccentricity).

Table 1. Experimental errors for different target diameters OD and eccentricities $r$

\begin{tabular}{|c|c|c|c|c|}
\hline OD $(\mathrm{mm})$ & $r(\mathrm{~mm})$ & $k$ & $\sigma$ theory $(\mathrm{mm})$ & $\sigma$ exp. $(\mathrm{mm})$ \\
\hline 3 & 0 & 1.1781 & 0 & N/A \\
\hline 3 & 0.3 & 1.1840 & 0.0002 & 0.0105 \\
\hline 3 & 0.6 & 1.2026 & 0.0020 & 0.0222 \\
\hline 3 & 0.9 & 1.2366 & 0.0061 & 0.0245 \\
\hline 3 & 1.2 & 1.2925 & 0.0162 & 0.0317 \\
\hline 3.3 & 0 & 1.2959 & 0 & N/A \\
\hline 3.3 & 0.3 & 1.3013 & 0.0003 & 0.0127 \\
\hline 3.3 & 0.6 & 1.3180 & 0.0025 & 0.0246 \\
\hline 3.3 & 0.9 & 1.3481 & 0.0075 & 0.0305 \\
\hline 3.3 & 1.2 & 1.3959 & 0.0203 & 0.0427 \\
\hline 3.6 & 0 & 1.4137 & 0 & N/A \\
\hline 3.6 & 0.3 & 1.4186 & 0.0003 & 0.0137 \\
\hline 3.6 & 0.6 & 1.4339 & 0.0030 & 0.0262 \\
\hline 3.6 & 0.9 & 1.4609 & 0.0094 & 0.0413 \\
\hline 3.6 & 1.2 & 1.5028 & 0.0264 & 0.0523 \\
\hline
\end{tabular}

\section{Conclusions}

This paper presents a measuring system using polymer-based 4Q CMUT array to detect circular target. The polymer-based CMUT provides advantages of low cost and large sensing area and is especially suit for detecting transparent objects. Experiments are conducted with three different target diameters and four different eccentricities to verify its feasibility. The dimension error is limited to $0.043 \mathrm{~mm}^{2}$ or $0.42 \%$ in area. The positioning error is $0.052 \mathrm{~mm}$ for target dimeter of 3.6 $\mathrm{mm}$ and eccentricity of $1.2 \mathrm{~mm}$. The 4Q CMUT array has less error if the target is closer to the center with less eccentricity. In this study, the largest target diameter is $60 \%$ of the 4Q CMUT array with maximum eccentricity before go outside the sensor. The relationship between target diameters and CMUT dimensions influence spatial resolution and range. The correcting algorithm affects inherited theoretical error. In the future, the optimized parameters of device design is suggested to improve for various position detection applications.

\section{Acknowledgments}

The authors would like to thank the financial support from the MEMS and Precision Machinery Research and Development Center at National Kaohsiung University of Applied Sciences for the development of the CMUTs. We would also like to acknowledge the Ministry of Sciences and Technology (MOST) of Taiwan (R.O.C.) for its partial support under project number MOST 1042221-E-151-012.

\section{References}

[1] M. I. Haller, B. T. Khuri-Yakub, A surface micromachined electrostatic ultrasonic air transducer, IEEE Trans. Ultrasonics, Ferroelectrics, Frequency Contr. 43(1) (1996) 1-6.

[2] B. T. Khuri Yakub, O. Oralkan, Capacitive micromachined ultrasonic transducers for medical imaging and therapy, NCBI, May, 21(5) (2011) 54004-54014. 
[3] M. M. W. Chang, M. T. Deng, J. T. Gwo, J. D. Mai, E. Hsu, 2006, January. Polymer-based capacitive micromachined ultrasonic transducers (cmut) for micro surgical imaging applications. In 2006 1st IEEE International Conference on Nano/Micro Engineered and Molecular Systems (pp. 61-65). IEEE.

[4] G. T. Bui, Y. Z. Chen, D. C. Pang, Polymer-based Capacity Micromachined Ultrasonic Transducer for Surface Roughness Measurement, Key Eng. Mater. 661 (2015) 22-28. Trans Tech Publications.

[5] Y. Wang, M. Gong, J. Ma, P. Yan, New radial tilt detection method using only one beam and one four-quadrant detector, Jpn. J. Appl. Phys. 43(11R) (2004) 7513.

[6] M. Toyoda, K. Araki, Y. Suzuki, Measurement of the characteristics of a quadrant avalanche photodiode and its application to a laser tracking system, Optic. Eng. 41(1) (2002) 145-149.

[7] J. D. Barchers, D. J. Lee, D. E. Lane, 1999, September. Single-input/single-output analysis of latency and quadrant detector saturation in adaptive optical systems. In SPIE's International Symposium on Optical Science, Engineering, and Instrumentation (pp. 308-320). International Society for Optics and Photonics.

[8] S. Olyaee, M. Rezazadeh, 3-D Measurement by dual four-quadrant position-sensitive detectors in the stereo mode. Int. J. Eng. Res. Appl. 2(3) (2012) 1157-1161.

[9] L. Dang, Z. Zhou, S. Tang, 2009, November. The characteristic analysis and optimization of quadrant detector signal. In Intelligent Computing and Intelligent Systems, 2009. ICIS 2009. IEEE International Conference on (Vol. 3, pp. 190-194). IEEE.

[10] E. B. Mohammed, M. Rehman, Digital capacitive angular-position sensor, IEE Proceed. Sci. Measurem. Technol. 150(1) (2003) 15-18. 